\title{
Qualidade e estilo de vida do paciente infartado: uma avaliação após a re-inserção social
}

\author{
Bruno Henrique Fiorin, M.Sc.*, Mirian Fioresi, D.Sc.**, Eliane de Fátima Almeida Lima***, \\ Lorena Barros Furieri, D.Sc. ${ }^{* * * *}$, Elizabete Regina Araújo de Oliveira, D.Sc.*****
}

${ }^{*}$ Enfermeiro, Hospital Santa Rita de Cássial UFES, ${ }^{* *}$ Enfermeira, Professora Adjunta do Departamento de EnfermagemUFES, ***Enfermeira, Doutoranda em Enfermagem - UFRJ, Professora Assistente do Departamento de Enfermagem-UFES, ****Enfermeira, Professora Adjunta do Departamento de Enfermagem-UFES, *****Enfermeira, Professora Titular do Departamento de Enfermagem-UFES

\section{Resumo}

Introduçâo: A melhoria da qualidade e do estilo de vida passou a ser um dos resultados esperados tanto das práticas assistenciais quanto das políticas públicas para o setor da promoção de saúde e da prevenção de doenças. Objetivo: Avaliar a qualidade e estilo de vida dos pacientes após o Infarto Agudo de Miocárdio (IAM) e desta forma traçar o perfil destes pacientes que são atendidos pela Atenção Básica de Saúde e os seus principais hábitos de vida. Material e métodos: Tratase de um estudo analítico, exploratório de corte transversal, realizado com pacientes que sofreram IAM, usuários do Sistema Único de Saúde (SUS), para coleta de dados utilizou a identificaçấo socioeconômica, instrumento de Qualidade de Vida - O Mac New QLMI e o instrumento de estilo de vida - Fantástico. Resultados e discussão: Foram estudados 23 pacientes nos quais $43,5 \%$ de pacientes do sexo masculino e $56,5 \%$ do sexo feminino. O histórico familiar e a presença de hipertensão arterial sistêmica estava presente em $95,7 \%$ da amostra. A qualidade de vida foi regular em $43,5 \%$ da amostra, ruim em $8,7 \%$, sendo apenas considerada excelente por $13,0 \%$. O estilo de vida foi alterado em $87 \%$ da amostra. $\mathrm{O}$ fato de possuir confiança em lidar com seus problemas apresentou uma forte correlação não somente com a classificação da QV, mas também mostrou impacto na determinação do estilo de vida. Conclusão: Apesar da melhora do estilo de vida, os pacientes apresentaram piora na qualidade de vida.

Palavras-chaves: qualidade de vida, estilo de vida, infarto do miocárdio, atenção primária a saúde.

\section{Abstract}

\section{Quality and style of life of infarcted patient: evaluation after social reinsertion}

Introduction: Improving the quality and style of life has become one of the outcomes of both care practices and public policies for health promotion and disease prevention. Objective: To assess the quality and style of life of patients after Acute Heart Attack (AHA) and thus trace the profile of these patients who are treated by primary care and its life habits. Methods: This is an analytical, exploratory cross-sectional study, conducted with patients who suffered AHA and 
were users of the Unified Health System (SUS), to collect data we used socioeconomic identification, Quality of Life Instrument - The Mac New QLMI instrument and Lifestyle-Fantastic. Results and discussion: We studied 23 patients in which $43.5 \%$ were male and $56.5 \%$ were female. The Family History and presence of systemic hypertension was present in $95.7 \%$ of the sample. Quality of life was regular in $43.5 \%$ of the sample, poor in $8.7 \%$, and excellent in $13.0 \%$. The lifestyle has changed in $87 \%$ of the sample. The fact that they have confidence in dealing with their problems strongly correlated not only with the classification of QOL, but also had an impact in determining lifestyle. Conclusion: Despite the improvement of lifestyle, patients had poorer quality of life.

Key-words: Quality of life, lifestyle, myocardial infarction, primary health care

\section{Resumen}

\section{Calidad y estilo de vida del paciente infartado: una evaluación después de la reinserción social}

Introducción: La mejora de la calidad y el estilo de vida se ha convertido en uno de los resultados esperados tanto en las prácticas asistenciales como en las políticas públicas para el sector de promoción de la salud y prevención de enfermedades. Objetivo: Evaluar la calidad y el estilo de vida de pacientes post Infarto Agudo de Miocardio (IAM), y así trazar el perfil de los pacientes que son tratados por la Atención Primaria de Salud y sus principales hábitos de vida. Material e métodos: Se trata de un estudio analítico, exploratorio transversal realizado con pacientes que sufrieron un IAM, usuarios del Sistema Único de Salud (SUS). Para colectar datos, se utilizó la identificación socioeconómica, instrumento de Calidad de Vida - El Mac-New QLMI e instrumento de vida - Fantástico. Resultados y discusión: Fueron estudiados 23 pacientes de los cuales $43,5 \%$ varones y $56,5 \%$ mujeres. El historial familiar y la presencia de hipertensión arterial estuvieron presentes en el 95,7 \% de la muestra. La calidad de vida fue regular en el $43,5 \%$ de la muestra, el 8,7\% mala y excelente en un 13,0 \%. El estilo de vida fue alterado en $87 \%$ de la muestra. El hecho de tener confianza en el trato con sus problemas correlacionó fuertemente no sólo la clasificación de la calidad de vida, sino que también tuvo un impacto en la determinación de la forma de vida. Conclusión: A pesar de la mejora del estilo de vida, los pacientes presentaron una peor calidad de vida.

Palabras-clave: calidad de vida, estilo de vida, infarto de miocardio, atención primaria de salud.

\section{Introdução}

O conceito de Qualidade (QV) e Estilo de Vida (EV), bem como a preocupação pela investigação destes indicadores, pode ser considerado relativamente recente e decorrem, em parte, de novos paradigmas que têm influenciado as políticas e as práticas de saúde nas últimas décadas. A melhoria da qualidade e do estilo de vida passou a ser um dos resultados esperados tanto das práticas assistenciais quanto das políticas públicas para o setor da promoção de saúde e da prevenção de doenças [1].

O termo qualidade de vida é amplo e inclui uma variedade maior de condições que podem afetar a percepção do indivíduo; seus sentimentos e comportamentos relacionados com o funcionamento diário de seu organismo [2]. Trata-se de um conceito multidimensional que envolve o grau de satisfação atingido no âmbito físico, emocional e social que pode ser tratado do ponto de vista objetivo e subjetivo, em abordagens individuais ou coletivas [3].
O estilo de vida é caracterizado por padróes de comportamentos identificáveis que podem ter um efeito profundo na saúde da população e está relacionado com diversos aspectos que refletem as atitudes, os valores e as oportunidades de vida de cada indivíduo [4]. Sendo assim o estilo de vida engloba todas as características do dia-a-dia; as atitudes e comportamentos praticados. É um conceito novo e pouco explorado pela área das Ciências da Saúde [3,5].

Avaliar tais condiçóes em pessoas infartadas tem sido uma maneira de determinar o impacto do cuidado de saúde quando a cura não é possível. Sendo considerados importantes fatores de avaliação dos resultados dos procedimentos terapêuticos, não apenas nos aspectos relacionados à redução dos sintomas e prolongamento da vida, mas, também, no que se referem à sobrevida do doente e a sua percepção sobre a QV [5,6].

As doenças cardiovasculares são reponsáveis por grande parte da morbidade e mortalidade que há no mundo, sendo consideradas uma das maiores endemias que ocorreram no século passado, porém 
de grande prevalência em nossa atualidade. Apesar dos avanços na terapêutica dos últimos anos, estas doenças são consideradas a principal causa de mortalidade na população brasileira, sendo responsável por 35\% dos casos de óbitos [7].

O processo de transição demográfica e epidemiológica vem ocorrendo de forma acelerada no Brasil aumentando ainda mais a ameaça das condições crônicas. A população brasileira está cada vez tornando-se mais idosa devido ao aumento da expectativa de vida e mais propícia a desenvolver problemas cardiovasculares, sendo esta frente de atuação o principal desafio da saúde pública: desenvolver açóes que contemplem a promoção, prevenção e reabilitação dos pacientes com problemas cardíacos reduzindo a mortalidade e a morbidade por estas doenças [8-10].

Neste cenário nota-se o aumento rápido da incidência de problemas cardiovasculares, sendo manifestado frequentemente pelo Infarto Agudo do Miocárdio (IAM), formas instáveis de angina e a morte súbita. Sendo a explicação deste fato o aumento da expectativa de vida da populaçáo, permitindo maior tempo para as doenças começar a se manifestarem $[11,12]$.

Desta forma objetivou-se, com este estudo, avaliar a qualidade e estilo de vida dos pacientes após o IAM e desta forma traçar o perfil destes pacientes que são atendidos pela Atenção Básica de Saúde e os seus principais hábitos de vida, dando ênfase aos fatores de riscos para doenças cardiovasculares predominantes após IAM.

\section{Material e métodos}

Trata-se de um estudo analítico, exploratório de corte transversal, realizado com pacientes que sofreram IAM, usuários do Sistema Único de Saúde (SUS), atendidos na Unidade de Saúde da Família (USF) de Maruípe, Vitória-ES.

Participaram do estudo um total de 23 pacientes, frequentadores da USF, que aceitaram voluntariamente contribuir com a pesquisa. Sendo que pacientes do sexo masculino deveriam possuir mais de 45 anos e do sexo feminino mais de 55 anos. Esta linha de corte foi estabelecida através da preconização do Ministério da Saúde, que considera que pacientes acima destas idades encontram-se no grupo de risco para doenças cardiovasculares [13].

Para a formação da amostra, foram feitas reunióes com as equipes de Saúde da Família, onde foram enumerados pelas mesmas todos os pacientes que já sofreram IAM e que estavam na área de abrangência da USF.

Para coleta de dados utilizou-se como instrumento, um roteiro de entrevista composta de três partes: a primeira com dados de identificação socioeconômica, a segunda com a aplicaçáo do instrumento de Qualidade de Vida - O Mac New QLMI, e a terceira com a aplicação do instrumento de Estilo de vida- Fantástico. O período de coleta foi entre dezembro de 2010 e maio de 2011.

O MAC NEW QLMI é um instrumento específico de qualidade de vida para pacientes após IAM, foi validado para a língua portuguesa em 1999 por Benetti e Nahas, em Florianópolis. É um instrumento confiável correspondendo às características específicas da qualidade de vida $[14,15]$. Este é composto de 27 questóes, dividido com escore máximo de 7 e no mínimo de 1 para cada questão, sendo ainda dividido em três domínios: físico, social e emocional. Seu escore varia de 1 a 189, quanto maior o escore melhor é considerada a qualidade de vida.

O instrumento de "Estilo de Vida Fantástico" é um instrumento genérico sendo validado para a Língua Portuguesa em 2008 por Añez, Reis e Petroski apresentando adequada consistência interna e validade do construto, sendo recomendado sua utilização na Atenção Básica e em estudos epidemiológicos [4]. É um instrumento composto por 25 questôes divididas em 9 domínios: família e amigos, atividade física, nutrição, cigarro e droga, álcool, sono, cinto de segurança, estresse e sexo seguro, tipo de comportamento, introspecção e trabalho. A soma de todos os pontos permite chegar a um escore total que classifica os pacientes em cinco categorias: Excelente, Muito Bom, Bom, Regular, Necessita Melhorar; seu escore varia de 0 a 100.

Após a coleta, os dados foram digitados no programa Excel 2007 e, posteriormente, codificados e descritos utilizando o programa estatístico Statistical Package for the Social Sciences (SPSS) 19.0 para Windows. Utilizou-se estatística descritiva e análise bivariada com os testes de correlação de Pearson e Qui-quadrado, respeitando o tipo de variável estudada.

Vale ressaltar que os princípios éticos contidos na Resolução 196/96 do Conselho Nacional de Saúde que trata da pesquisa com seres humanos foram devidamente respeitados, garantindo-se o anonimato dos partícipes e sua saída da pesquisa 
quando considerasse necessário. Para participar do estudo o paciente deveria assinar o termo de Livro Consentimento e Esclarecido. Este estudo foi aprovado pelo Comitê de Ética e Pesquisa da Universidade Federal do Espírito Santo com parecer $n^{\circ} 20 / 2010$.

\section{Resultados}

Foram estudados 23 pacientes com histórico prévio de Infarto Agudo do Miocárdio. A amostra foi composta por $43,5 \%$ de pacientes do sexo masculino e $56,5 \%$ do sexo feminino.

Quanto à etnia declarada $56,5 \%$ se declararam de raça branca, 39,1 \% de raça parda e 4,3 \% negra. Houve frequência igualitária e predominante entre viúvos e casados na avaliaçáo do estado civil, ambos correspondem a 47,8 \% da amostra.

A escolaridade predominante foi o $2^{\circ}$ grau completo com $34,8 \%$. O analfabetismo esteve presente em 17,4\% e nenhum paciente da amostra possui o ensino superior completo, conforme ilustra a Tabela I.

Tabela I - Caracterização da amostra estudada.

\begin{tabular}{|c|c|c|c|}
\hline & & Pacientes & $\begin{array}{c}\text { Porcenta- } \\
\text { gem }\end{array}$ \\
\hline \multicolumn{4}{|l|}{ Sexo } \\
\hline & Masculino & 10 & 43,5 \\
\hline & Feminino & 13 & 56,5 \\
\hline \multicolumn{4}{|c|}{ Estado civil } \\
\hline & Casado & 11 & 47,8 \\
\hline & Viúvo & 11 & 47,8 \\
\hline & Divorciado & 1 & 4,3 \\
\hline \multicolumn{4}{|c|}{ Escolaridade } \\
\hline & Analfabeto & 4 & 17,4 \\
\hline & $1^{\circ}$ grau incompleto & 5 & 21,7 \\
\hline & $2^{\circ}$ grau incompleto & 3 & 13 \\
\hline & $2^{\circ}$ grau completo & 8 & 34,8 \\
\hline & Superior incompleto & 3 & 13 \\
\hline Total & & 23 & $100 \%$ \\
\hline
\end{tabular}

A idade da amostra variou de 55 a 86 anos de idade, com idade média de 68,17 e desvio padrão de 8,5 . Sendo a faixa etária de 65 a 69 anos a de maior prevalência no estudo com $30,4 \%$ dos pacientes, seguido da faixa etária de 55 a 59 anos com 21,7 $\%$. Nas faixas etárias de 60 a 64, 70 a 74 e 80 a 79 tivemos distribuição igualitária.

A renda mensal declarada variou de $\mathrm{R} \$ 400,00$ a 3500,00 tendo como media $\mathrm{R} \$ 1461,67$. A maioria da população $(38,1 \%)$ possui renda compreendi- da de $\mathrm{R} \$ 545,00$ e $\mathrm{R} \$ 1090,00.8,7 \%$ vivem com renda menor de $\mathrm{R} \$ 545,00$ e $17,4 \%$ possui renda mensal superior a $\mathrm{R} \$ 2180.78,2 \%$ dos pacientes estudados são aposentados e o restante $(21,8 \%)$ possui vínculo empregatício ativo.

O tempo após o IAM variou de 1 a 15 anos, sendo o tempo médio de seis anos e desvio padrão de 3,8. Dentre os fatores de riscos enumerados, o histórico familiar foi unânime acompanhado da Hipertensão Arterial Sistêmica (HAS), que estava presente em 95,7\% da amostra, ou seja, em 22 pacientes. As dislipidemias estiveram presentes em $44,5 \%$, enquanto o tabagismo é o menos frequente $(4,3 \%)$. A distribuiçáo dos fatores de riscos pode ser observada na Figura 1.

Figura 1 - Representação da presença de fatores de riscos para doenças cardiovasculares.

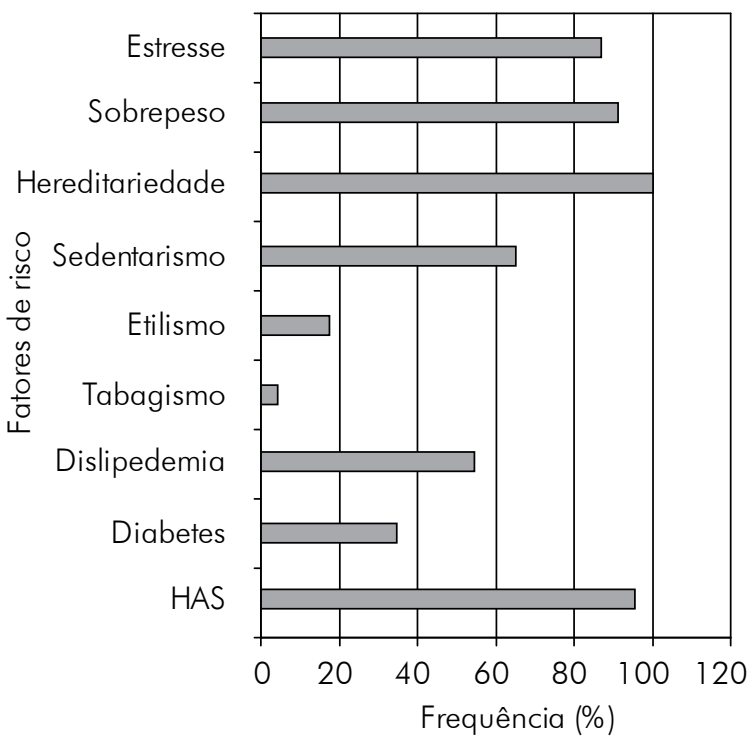

Apesar da frequência de etilismo e tabagismo serem relativamente baixa no estudo, $43,5 \%$ da amostra afirmaram que possuía histórico tanto de etílismo e tabagismo antes do IAM, e que estes hábitos foram modificados após a doença.

Além da maioria dos pacientes se declarar sedentário e estressado, os hábitos de lazer não foram frequentes na população, sendo algo eventual, $69,5 \%$ da amostra declarou realizar atividades de lazer, enquanto $4,3 \%$ e $8,9 \%$ declararam realizar atividades de lazer diariamente e semanalmente respectivamente. Houve relação estatística entre os casos de HAS e a baixa frequência da prática de lazer $(\mathrm{p}<0,01)$, mostrando que a prática constante do lazer pode se influenciar na prevalência de HAS na amostra estudada. 
O local de assistência e tratamento foi de 43,5 $\%$ em instituição pública, 17,4 \% em instituiçôes particulares e $39,1 \%$ dos pacientes relataram fazer acompanhamento tanto em instituiçóes públicas quanto privadas. Um quantitativo de $65,2 \%$ da amostra afirmou que o SUS náo consegue prestar toda a assistência necessária ao paciente infartado, porém $100 \%$ da populaçáo declaram ir a USF ao menos uma vez por mês para consulta e acompanhamento terapêutico.

O escore médio da qualidade de vida observado foi de 126,3, sendo a variação de 66 a 167. A qualidade de vida pode ser considerada regular em 43,5\% da amostra, ruim em 8,7\%, sendo apenas considerada excelente por 13,0 \% como se observa na Tabela II. Ao questionar sobre o impacto do IAM sobre a qualidade de vida, 65,2 $\%$ dos pacientes afirmaram que houve piora, 21,7 $\%$ que houve melhora e $13 \%$ náo perceberam alteração.

Tabela II - Distribuição da classificação da qualidade de vida, utilizando o questionário "QLMI Mac New".

\begin{tabular}{lcc}
\hline \multicolumn{1}{c}{ Qualidade de Vida } & Frequência & Porcentagem \\
\hline Excelente & 3 & 13,0 \\
Boa & 8 & 34,8 \\
Regular & 10 & 43,5 \\
Ruim & 2 & 8,7 \\
Total & 23 & 100 \\
\hline
\end{tabular}

Houve correlação negativa entre o fato de se sentir inútil e deslocado do ambiente em relação à classificaçáo da QV, enquanto que o sentimento de confiança e a capacidade de lidar com o problema cardíaco apresentaram uma correlação positiva ( $\mathrm{p}$ $<0,0001)$. Pode-se inferir também que a limitação física, bem como vertigem e dores na perna também apresentam uma correlação negativa com a determinação da QV ( $\mathrm{p}<0,001)$. Estes dados estão contidos na Tabela III.

O estilo de vida foi alterado em $87 \%$ da amostra, conforme afirmação dos pacientes. $\mathrm{Na}$ populaçáo estudada o escore do EV variou de 47 a 78, possuindo o escore médio de 64,78. A maioria apresentou um estilo de vida considerado "Bom" e "Muito Bom", a frequência de classificação do estilo de vida baseado no instrumento "Estilo de Vida Fantástico" pode ser observado na Tabela IV.
Tabela III - Fatores que influenciam na determinação da QV determinada através da correlação de Pearson.

\begin{tabular}{lcc}
\hline Fator que influencia na QV & $\begin{array}{c}\text { Correla- } \\
\text { ção }\end{array}$ & $\begin{array}{c}\text { Significância } \\
\text { (p-valor) }\end{array}$ \\
\hline Sentir inútil e deslocado & $-0,853$ & 0,00001 \\
Ter confiança para lidar & 0,823 & 0,00001 \\
com os problemas & $-0,645$ & 0,01 \\
Sentir desencorajado & $-0,68$ & 0,00001 \\
Sentir dependente & $-0,716$ & 0,00001 \\
Dor no Peito & $-0,716$ & 0,00001 \\
Dor na Perna & $-0,639$ & 0,01 \\
Apreensão e medo & $-0,712$ & 0,001 \\
Vertigem & $-0,656$ & 0,001 \\
Familiares superprotetores & $-0,726$ & 0,001 \\
Exclusão de atividades & $-0,629$ & 0,01 \\
Limitações em realizar & & \\
atividades físicas & $-0,658$ & 0,01 \\
Afetação nas relações & & \\
sexuais & & \\
\hline
\end{tabular}

Tabela IV - Distribuição da classificação do estilo de vida da amostra estudada, utilizando o questionário de "Estilo de Vida Fantástico".

\begin{tabular}{lcc}
\hline Estilo de Vida & Frequência & Porcentagem \\
\hline Muito bom & 9 & 39,1 \\
Bom & 11 & 47,8 \\
Regular & 3 & 13 \\
Total & 23 & 100 \\
\hline
\end{tabular}

O principal fator que influencia no estilo de vida é o fato de ter alguém para conversar $(\mathrm{p}=0,02)$. $\mathrm{O}$ fato de sentir-se inútil e deslocado apresenta uma correlação negativa de 0,658 com a classificação do estilo de vida ( $p=0,01)$, assim como o fato de sentir-se agitado e nervoso, que apresenta uma correlaçáo de 0,668 ( $\mathrm{p}<0,0001)$. O sentimento de confiança e segurança apresenta uma correlaçáo positiva de $0,649(\mathrm{p}=0,01)$ em relaçáo à classificação do EV.

\section{Discussão}

A população avaliada foi composta por 23 pacientes sendo sua maioria do sexo feminino (56,5\%). Este dado é diferente de vários estudos no qual a população predominante acometida é do sexo masculino, uma vez que é considerada mais propícia ao IAM por apresentarem maior número de fatores de risco $[1,2,12,14]$.

Este fato pode ser explicado através de recentes estudos que mostram que doenças cardíacas, sobretudo coronarianas, apresentam um aumento elevado de incidência em mulheres após a menopausa. Isto se deter- 
mina pelas alterações hormonais e pelas transformaçóes sociais e culturais que estimulam a maior participação da mulher no mercado de trabalho e a progressiva adoção de hábitos nocivos à saúde [2]. Este fato também pode ser explicado pela taxa de mortalidade elevada do gênero masculino quando comparado aos do gênero feminino no que decorre ao acometimento e agravos pela doença cardiovascular [7,9].

A idade da amostra variou de 55 a 86 anos de idade, com idade média de 68,17. A faixa etária predominante foi de 65 a 69. Dados similares foram encontrados no INTERHEART: estudo Latino Americano, que investigava os principais fatores de risco para IAM na América Latina [12], assim como em estudos nacionais $[16,17]$. É notório que a cada ano maior se torna à longevidade da populaçáo. Este fato pode ser correlacionado ao aumento de conhecimento sobre a carga das doenças e na eficácia dos tratamentos [10].

Os fatores de riscos predominantes, encontrados em nosso estudo, como mostra a figura 1, foram a história familiar de IAM e a HAS. Sabe-se que os fatores genéticos apresentam uma correlação positiva com as condições de disfunçóes cardiovasculares e no agravamento das comorbidades que surgem no processo de adoecimento. $\mathrm{O}$ histórico familiar é considerado um fator de risco não modificável, por isso indivíduos com este fator de risco precisam ser acompanhados e orientados, quanto às práticas de vida saudável na tentativa de minimizar o impacto de outros fatores de risco [18].

95,7\% da amostra eram hipertensos e estavam em tratamento contínuo. Estes dados foram superiores aos encontrados pelo estudo FRICAS [19] e pelo INTERHEART [12], porém se assemelha a um estudo realizado em Santa Catarina, onde se objetivou avaliar os fatores de risco cardiovasculares em paciente com doença aterosclerótica através de um estudo do tipo caso controle [20].

A HAS é um fator de risco importante para o IAM e sua permanência determina um pior prognóstico após IAM, tanto a curto como em longo prazo. No Brasil é a doença mais prevalente no adulto [21] e a sua relação com as doenças cardiovasculares é progressiva, sendo independente de outros fatores de risco. A HAS eleva a incidência de acidentes vasculares encefálicos (AVE), bem como outras doenças cardiovasculares após o IAM [17,20,22,23].

Após o IAM muitos pacientes apresentam dificuldade para voltar a realizar suas atividades de vida diária e este fato pode justificar um elevado percentual de sedentarismo na amostra. Dentre os 23 pacientes, $65,2 \%$ não praticavam nenhuma atividade física durante a semana. O sedentarismo, muitas vezes, está relacionado ao comportamento cotidiano decorrente dos confortos da vida moderna, este, por sua vez impulsiona o surgimento de problemas cardiovasculares e influência nas condiçóes de obesidade, sobrepeso, dislipidemias e diabetes [24,25].

Além disso, esta dificuldade também pode estar relacionada com a baixa prevalência de prática frequente de momentos de lazer. Notamos que a população estudada afirma realizar de modo eventual ou nunca praticá-lo conforme a figura 4 , sendo este um grande prejuízo para a saúde dos indivíduos, uma vez que se nota uma correlação negativa entre a prática de lazer frequente e os casos de HAS. Desta forma notamos que medidas simples, que favorecem a prática de atividade física e de lazer podem influenciar no tratamento de doenças, tendo impacto direto sobre a concepção de qualidade de vida.

Em relação ao tabagismo, como demonstra a figura 1, apenas $4,3 \%$ declaram ser tabagistas com hábito diário de fumar. No entanto, não se pode deixar de citar que $43,5 \%$ dos participantes relataram ser etilistas e tabagistas antes do IAM, tendo hábito modificado após o IAM. O tabagismo de forma geral é um grande agravante de doenças cardiovasculares, devido às alteraçóes metabólicas geradas na hemodinâmica do organismo [26].

O tabagismo é um fator de risco que, estatisticamente, se distribui homogeneamente na América do Sul, bem como, apresenta chance similar de gerar um ataque cardíaco similar entre os paises como mostra o estudo [12]. Em um estudo longitudinal de duração aproximada de 12 anos, onde se objetivava analisar a correlação do tabagismo com os outros fatores de risco para IAM, Prescott e col. [27] demonstraram que este é o fator de risco mais passível de modificação. Afirma, ainda, que as mulheres tabagistas apresentam maiores chances de sofrer IAM do que os homens, especialmente nas idades acima de 55 anos, em que esta chance excede a $50 \%$ quando comparado ao sexo masculino. No entanto, em homens que, além de serem tabagistas, apresentam consumo de álcool e pouca prática de atividades física, este valor aumenta, passando ser o grupo mais vulnerável ao IAM.

A manutenção destes e outros fatores de risco são preocupantes, pois potencializa as comorbidades oriundas do IAM, bem como novos problemas cardiovasculares. A maioria dos pacientes, como mostra a Tabela IV, apresenta mais de três fatores de risco, dados similares foram encontrados por Colombo e Aguillar [28] que avaliaram o estilo de vida após IAM. 
Lane [29] mostra que a taxa de mortalidade nos pacientes após IAM, nos primeiros 12 meses é de 10,8 \% e após 18 meses esta taxa reduz para 8,6 \%, sendo que tais valores podem ainda ser maiores, caso náo seja feito o controle e acompanhamento destes pacientes. As principais causas de óbitos apontadas foram a falência cardíaca e arritmias por recorrência do IAM.

A avaliação do estilo de vida mostrou que os hábitos seguidos pelos pacientes apresentam, em sua maioria, a classificação de "Bom" e "Muito Bom", após avaliaçáo usando instrumento de "Estilo de Vida Fantástico". Esta classificação esta condizente com as afirmativas de $87,0 \%$ da amostra, que relata ter seu estilo de vida melhorado após IAM, para se adequar ao tratamento. Porém, mesmo com estas alteraçóes nota-se que ainda há manutenção dos fatores de risco cardiovasculares como discutido anteriormente, desta forma corrobora-se Colombo \& Aguiar [28] afirmando que apesar dos infartados apresentarem uma classificação do estilo de vida "boa" o grupo estudado possui hábitos que propiciam a manutenção ou progressão de coronariopatias.

A qualidade de vida pode ser entendida como a concepção pessoal de cada indivíduo sobre suas condiçôes de vida. Este indicador torna-se cada vez mais valorizado para a avaliaçáo de tecnologias e terapêuticas utilizadas na área da saúde. Para a sua mensuração vêm sendo criados e validados instrumentos específicos para cada patologia, como é o caso do QLMI Mac New em relação ao IAM [30].

Na concepção da população estudada, a maioria afirmou que houve piora em sua qualidade de vida após o IAM. Esta informaçáo confirma os dados apresentados após aplicar o questionário específico de QV, no qual 43,5 \% e 8,7 \% dos pacientes apresentaram uma qualidade de vida "regular" e "ruim", respectivamente (Tabela II). Estes dados corroboram os dados apresentados em algumas dissertaçóes e teses que fizeram uso do mesmo instrumento com metodologia similar [16,17,31].

A análise da qualidade de vida neste estudo vai ao encontro com o estudo realizado por Gois e colaboradores [1]. Estes autores avaliaram a qualidade de vida dos pacientes antes e após seis meses da cirurgia de revascularizaçáo e concluíram que a QV de vida melhorou após o procedimento em praticamente todos os aspectos que tangem esta dimensão da saúde.

Ao realizar a correlação de Pearson notamos que a maioria das variáveis que apresentaram significância com o escore de classificação da QV se mostraram com uma correlação negativa conforme ilustrado na Tabela III. O fato de possuir confiança em lidar com seus problemas apresentou uma forte correlação não somente com a classificação da QV, mas também mostrou impacto na determinação do estilo de vida. Desta forma notamos a importância de se trabalhar com os aspectos emocionais dos pacientes após IAM $[1,5,6]$.

O sentimento de ser inútil e deslocado mostrou-se maior correlação negativa em relação à classificação da $\mathrm{QV}$, desta forma quanto mais o paciente apresentar estes sentimentos pior vai ser sua concepção de QV. Seguindo este mesmo padrão sintomas como dores na perna, no peito, vertigens também apresentam impactos sobre a condição de saúde dos clientes, aspectos estes que precisam ser trabalhados durante a terapêutica. Brown [32] descreve dados similares em que a dificuldade de retornar as atividades comuns diárias, o retorno ao trabalho, a experiência das sintomatologias após IAM bem como as vivências de comorbidades apresentam impactos negativos sobre a concepção de QV pelos pacientes em longo prazo, sendo mais impactantes para pacientes mais jovens.

O lazer, como forma de interação social, novas amizades, o apoio das pessoas queridas e a não superproteçáo faz como que o paciente se sinta melhor. O IAM, de certa forma, não acomete somente o paciente, mas toda a família e pessoas próximas, e quando essas pessoas estão presentes, dando apoio, as angústias, medos e inquietaçóes são compartilhadas e diluídas $[2,14]$.

Frente ao exposto, torna-se necessária implementação de programas de reabilitação após IAM, que incentivem os pacientes a reconhecerem e trabalharem as limitações atribuídas ao seu novo estado de saúde. Ainda é importante que estes programas não só envolvam os infartados, mas que englobem toda a sua família, conscientizando a mesma para este processo de reabilitação e re-inserção do familiar acometido.

\section{Conclusão}

Conclui-se, com este estudo, que os pacientes avaliados após o IAM, apesar de relatarem mudanças nos hábitos e no estilo de vida apresentaram piora no quesito qualidade de vida. Sendo preocupante a elevada prevalência de vários fatores de riscos para doenças cardiovasculares nesta população alertando a necessidade de políticas públicas de saúde, específicas neste processo de reabilitação e re-inserção social. 


\section{Referências}

1. Gois CFL, Dantas RAS, Torrati FG. Qualidade de vida relacionada à saúde antes e seis meses após a revascularizaçáo do miocárdio. Rev Gaúcha Enferm 2009;30(4):700-7.

2. Caetano JA, Soares E. Qualidade de vida de clientes pós-infarto agudo do miocárdio. Esc Anna Nery 2007;11(1):30-37.

3. Minayo MCS, Hartz Z MA, Buss PM. Qualidade de vida e saúde: um debate necessário. Ciênc Saúde Coletiva 2000;5(1):7-18.

4. Anez RCR, Reis RS, Petroski EL. Versão brasileira do questionário "estilo de vida fantástico": tradução e validação para adultos jovens. Arq Bras Cardiol 2008;91(2):102-9.

5. Dantas RAS, Góis CFL, Silva LM. Utilização da versáo adaptada da escala de qualidade de vida de Flanagan em pacientes cardíacos. Rev Latinoam Enferm 2005;13(1):15-20.

6. Dantas RAS, Rossi LA, Costa MCS, Vila VSC. Qualidade de vida após revascularização do miocárdio: avaliaçâo segundo duas perspectivas metodológicas. Acta Paul Enferm 2010;23(2):163-8.

7. Thomas CV, Souza AL, Portal VL, Ruschel P P. Avaliação Ambulatorial de qualidade de vida em pacientes pós-infarto agudo do miocárdio. Revista SBPH 2007;10(2):56-65.

8. Silva Junior AB, Oliveira IM, Alcântara EC, Resende ES. Fatores de risco para síndromes coronarianas e descrição dos questionários de vida Mac new QLMI e SF-36. Arq Ciênc Saúde UNIPAR 2006;10(1):49-54.

9. Schmidt MI. Doenças crônicas não transmissíveis no Brasil: carga e desafios atuais. Saúde no Brasil 4. The Lancet 2011;61-74.

10. Mendes EV. Capítulo 1 - As situações das condições de saúde e os sistemas de atençáo à saúde. As redes de atenção à saúde. Brasília: Organização Pan-Americana da Saúde; 2011.

11. Silva Junior AB, Oliveira IM, Alcântara EC, Resende ES. Fatores de risco para síndromes coronarianas e descrição dos questionários de vida Mac New QLMI e SF-36. Arq Ciênc Saúde UNIPAR 2006;10(1):49-54.

12. Lanas F, Avezum A, Bautista LE, Diaz R, Luna M, Islam $S$ et al. Risk factor acute myocardial infarction in Latin America: The INTERHEART Latin American Study. Rev Bras Hipertens 2007;14(4):278-9.

13. Ministério da Saúde. Prevençáo Clínica de Doença Cardiovascular, Cerebrovascular e Renal Crônica: Cadernos de Atenção Básica - n. ${ }^{\circ}$ 14. Brasília: Ministério da Saúde; 2006.

14. Oldridge N, Guyatt G, Crowe J, Feeny D, Jones N. Goal attainment in a randomized controlled trial of rehabilitation after myocardial infarction. J Cardiopulm Rehabil 1999;19(1):29-34.

15. Benetti M, Nahas MV, Barros MVG. Reproducibility and validity of a Brazilian version of the Mac New quality of life after myocardial infarction (Mac New QLMI) questionnaire. Medicine \& Science in Sports \& Exercise 2001;33(5).

16. Sivieiro IMPS. Saúde Mental e qualidade de vida de enfartado [tese]. Ribeirão Preto: Universidade de São Paulo; 2003.
17. Alcântara EC. Qualidade de vida após infarto agudo do miocárdio: avaliaçáo com os questionários Mac New QLMI e SF-36 [dissertação]. Uberlândia: Universidade Federal de Uberlândia; 2006.

18. Fermino RC, Seabra A, Garganta R, Maia JAR. Fatores genéticos na agregação familiar da pressão arterial de famílias nucleares portuguesas. Arq Bras Cardiol 2009;92(3):209-215.

19. Silva MAD, Sousa AGMR, Schargodsky H. Fatores de risco para infarto do miocárdio no Brasil: estudo FRICAS. Arq Bras Cardiol 1998;71(5):667-75.

20. Alves FMB, Cosentino MB, Sakae TM. Fatores de risco cardiovascular em pacientes com doença aterosclerótica não coronariana em hospital no Sul do Brasil. Estudo caso-controle. Rev Bras Clin Méd 2009;7:3-10.

21. Favarato MECS, Favarato D, Hueb WA, Aldrighi JM. Qualidade de vida em portadores de doença arterial coronária: comparação entre gêneros. Rev Assoc Med Bras 2006;52(4):236-41.

22. Chobanian AV. Seventh report of the joint national committee on prevention, detection, evaluation, and treatment of high blood pressure: the JNC 7 report. Hypertension 2003;289:2560-72.

23. Oliveira DC. Fatores de risco para acidente vascular encefálico após cirurgia de revascularizaçâo do miocárdio. Arq Bras Cardiol 2008;91(4):234-7.

24. Rezende FAC, Rosado LEFPL, Ribeiro RCL, Vidigal FC, Vasques ACJ, Bonard IS, et al. Índice de massa corporal e circunferência abdominal: associação com fatores de risco cardiovascular. Arq Bral Cardiol 2006;87(6):728-34.

25. Melchiors AC, Cassyano JC, Roberto P, Santos FOS, Souza PRA, et al. Qualidade de vida em pacientes hipertensos e validade concorrente do Minichal-Brasil. Arq Bras Cardiol 2010;94(3):357-64.

26. Nogueira CRSR, Hueb W, Takiuti ME, Girardi PBMA, Teryo N, Fernandes F, et al. Qualidade de vida após revascularização cirúrgica do miocárdio com e sem circulação extracorpórea. Arq Bras Cardiol 2008;91(4):238-44.

27. Prescott E, Hippe M, Schnohr P, Hein HO. Smoking and risk of myocardial infarction in women and men: longetudinal population study. BMJ 1998;316(4):1043-47.

28. Colombo RCR, Aguillar OM. Estilo de vida e fatores de risco de pacientes com primeiro episódio de infarto agudo do miocárdio. Rev Latinoam Enferm 1997;5(2):69-82.

29. Lane D, Carroll D, Ring C, Beevers DG, Lip GY. Mortality and Quality of the life 12 months after myocardial infarction: effects of depression and anxiety. Psychosomatic Medicice 2001;63:221-30.

30. Thompson DR, Yu CM. Quality of life in patients with coronary heart disease-I: Assessment tools. Health Qual Life Outcomes 2003;1(42):123-30.

31. Vasconcelos CB. Qualidade de vida, ansiedade e depressão após infarto agudo do miocárdio [dissertação]. Uberlândia: Universidade Federal de Uberlândia; 2007.

32. Brown N, Melville M, Gray D, Young T, Munro J, Skene A M. Quality of life four years after acute myocardial infarction: short form 36 scores compared with a normal population. Heart 1999;81(4):352-8. 